\title{
Inhalt/Contents 10/01
}

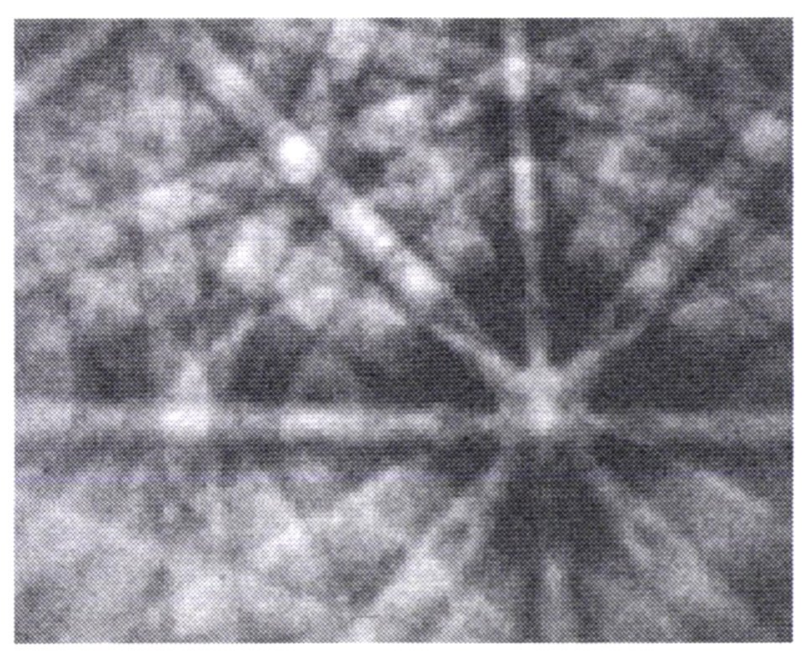

\section{Katrakova, F. Mücklich}

Probenpräparation für die Rückstreuelektronen-Kikuchi-Beugung (Electron Backscatter Diffraction, EBSD)

Specimen Preparation for Electron Backscatter Diffraction Part I: Metals

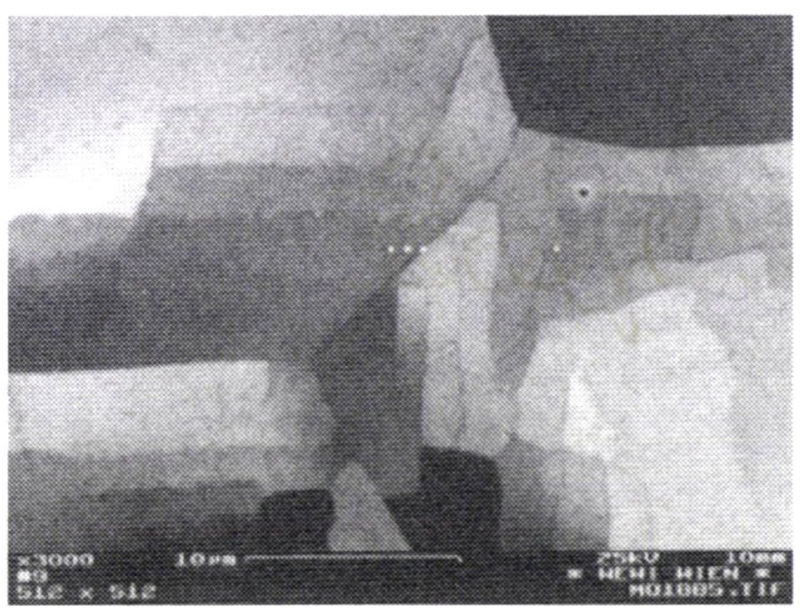

\section{Stickler}

REM-ECC Abbildungen und SAC-Diagramme - Verfahren zur zerstörungsfreien Gefügecharakterisierung und Ermittlung der globalen Versetzungsanordnung

SEM-ECC Imaging and SAC-Patterns - Procedures for the nondestructive characterization of microstructures and for revealing the global dislocation arrangement

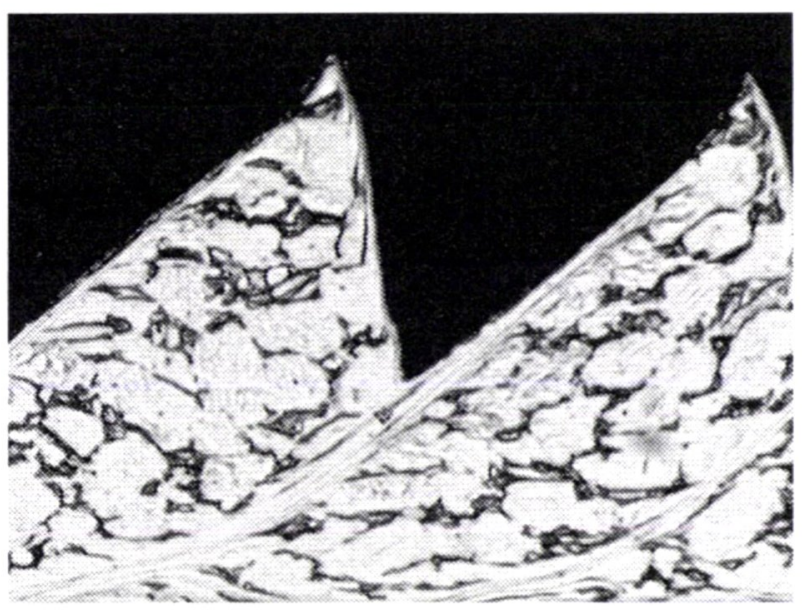

\section{Siemers, M. Bäker, C. Grusewski, D. Mukherji, J. Rösler}

Querschnittpräparation von Spänen aus Ti6Al4V für die Analyse im Transmissionselektronenmikroskop - Verfahren und erste Ergebnisse

Cross-sectional TEM Sample Preparation for the Analysis of Chips of Ti6Al4V - Preparation Method and a few Results

- Mitteilungen/Information

- Heitere Metallographie/Humorous Metallography

- Veranstaltungskalender/Meeting Diary

th Vorschau auf das nächste Heft unter:

Dreview on next issue at: 


\section{Praktische Metallographie \\ Practical Metallography}

\section{Gründer/Founders}

Günter Petzow

Gerhard Reinacher $†$

Charlotte Wachau $†$

\section{Herausgeber/Editor}

Prof. Dr. G. Petzow

Schriftleiter

Editor in charge

Prof. Dr.-Ing. F. Mücklich

Universität des Saarlandes

Lehrstuhl für Funktionswerkstoffe

Postfach 151150

D-66041 Saarbrücken

Telefon: +49/681 302-2048

Telefax: +49/681 302-4876

E-Mail: pm-editor@matsci.uni-sb.de

\section{Redaktion / Editor}

Dipl.-Ing. S. Mücklich

Zöblitzer Str. 10

D-09125 Chemnitz

Telefon: +49/371/531-5384

Telefax: +49/371/531-6179

E-Mail: silke.muecklich@mbv.tuchemnitz.de

\section{Wissenschaftlicher Beirat/Editorial Board}

Dr. C. Bagnall, MCS Associates Inc., Greensburg, PA (USA)

Dr. E. Bischoff, MPI für Metallforschung, Stuttgart

C. Bochert, Wirtz-Buehler GmbH, Düsseldorf

Prof. Dr. H.-E. Bühler, RWTH Aachen

V. Dietl, Lette Verein Berlin

Dr. G. Elssner, MPI für Metallforschung, Stuttgart

Prof. Dr. H.E. Exner, TU Darmstadt

Dr. M. Göken, Univ. des Saarlandes, Saarbrücken

Prof. Dr. E. Hornbogen, Ruhr-Universität Bochum

Prof. Dr. F. Jeglitsch, Montanuniversität Leoben

Dr. H.-J. Klaar, RWTH Aachen

Prof. Dr. A. KneissI, Montanuniversität Leoben

Dr. W.-U. Kopp, Daisendorf

Dr. J. Paul, Leica Vertrieb GmbH, Bensheim

Prof. Dr. M. Pohl, Ruhr-Universität Bochum

Prof. Dr. Schmitt-Thomas, IST, München

Prof. Dr. G. Schneider, Bosch, Stuttgart

U. Täffner, MPI für Metallforschung, Stuttgart

Dr. J. Trempler, Martin-Luther-Univ. Halle-Wittenberg

Prof. Dr. H.-H. Uchida, Tokai Univ., Hiratsuka, Kanagawa

G. Vander Voort, Buehler Ltd., Illinois

Prof. Dr. B Wielage, TU Chemnitz

Dr. H.-J. Wieland, Verein Dt. Eisenhüttenleute, Düsseldorf

\section{Eingebettete Proben obne Aufwand! SIMPLIMET ${ }^{\circledR}$ Einbettpressen von BUEHLER ${ }^{\circledR}$}

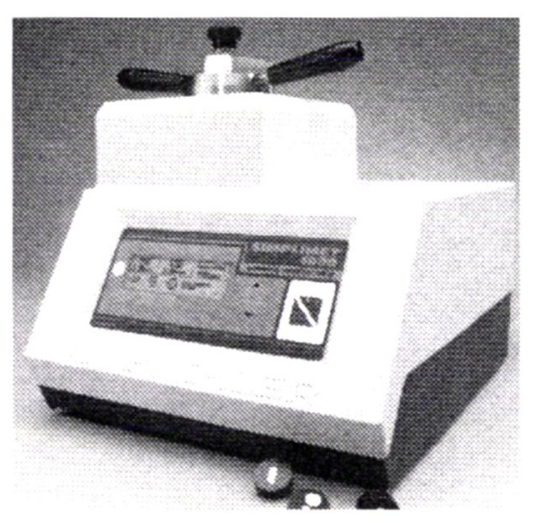

SIMPLIMET ${ }^{\circledR} 1000$ setzt neue Maßstäbe beim Einbetten Ihrer Proben. Der Druck wird elektrohydraulisch aufgebracht, das Einbringen Ihrer Muskelkraft oder Druckluft entfällt. Zum Betrieb benötigen Sie $40 \mathrm{~cm}$ Tischfläche, eine Steckdose und Wasser. Nach dem Bestücken der Presse läuft der Einbettvorgang automatisch ab und Sie können sich wichtigeren Dingen im Labor widmen.

\section{Kompetenz in Materialographie und Werkstoffprüfung}

\section{Bei Präparationsproblemen stehen Ihnen unsere Anwendungslabors zur Verfügung}

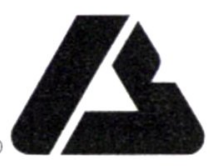

BUEHLER
Buehler GmbH • In der Steele 2 • D-40599 Düsseldorf • Tel. 0211/974100 • Fax 0211/9741079 Ing. Andreas Grimas • Hauptstr. 3 • A-3012 Wolfsgraben • Tel. 02233/78610 • Fax 02233/78619 Prüfmaschinen AG • Postfach • CH-8953 Dietikon 1 · Tel. 01/7464030 • Fax 01/7464039 e-mail:vertrieb@buehler-met.de •http://www.buehler-met.de•http://www.buehler.com

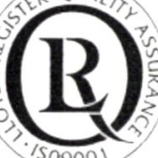

Reg.-Nr. 207017 